\title{
Supramolecular Nanostructure Formation of Coassembled Amyloid Inspired Peptides
}

\author{
Goksu Cinar, ${ }^{\dagger}$ Ilghar Orujalipoor, ${ }^{\ddagger}$ Chun-Jen Su, ${ }^{\S}$ U-Ser Jeng, ${ }^{\S}$ Semra Ide, ${ }^{\ddagger}, \|$ and Mustafa O. Guler* ${ }^{\dagger}$ \\ ${ }^{\dagger}$ Institute of Materials Science and Nanotechnology, National Nanotechnology Research Center (UNAM), Bilkent University, \\ 06800 Ankara, Turkey \\ ${ }^{*}$ Department of Nanotechnology and Nanoscience, Hacettepe University, 06800 Beytepe, Ankara, Turkey \\ ${ }^{\S}$ National Synchrotron Radiation Research Center, 101 Hsin-Ann Road, Hsinchu Park, Hsinchu, Taiwan \\ "Department of Physics Engineering, Hacettepe University, 06800 Beytepe, Ankara, Turkey
}

\section{Supporting Information}

ABSTRACT: Characterization of amyloid-like aggregates through converging approaches can yield deeper understanding of their complex self-assembly mechanisms and the nature of their strong mechanical stability, which may in turn contribute to the design of novel supramolecular peptide nanostructures as functional materials. In this study, we investigated the coassembly kinetics of oppositely charged short amyloidinspired peptides (AIPs) into supramolecular nanostructures by using confocal fluorescence imaging of thioflavin $\mathrm{T}$ binding, turbidity assay and in situ small-angle X-ray scattering (SAXS)

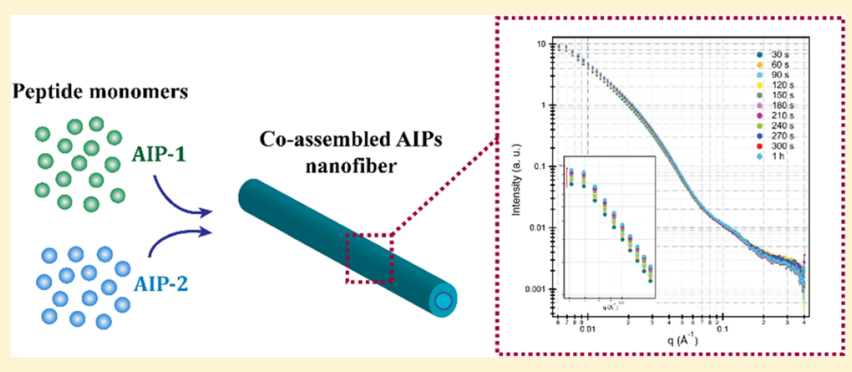
analysis. We showed that coassembly kinetics of the AIP nanostructures were consistent with nucleation-dependent amyloid-like aggregation, and aggregation behavior of the AIPs was affected by the initial monomer concentration and sonication. Moreover, SAXS analysis was performed to gain structural information on the size, shape, electron density, and internal organization of the coassembled AIP nanostructures. The scattering data of the coassembled AIP nanostructures were best fitted into to a combination of polydisperse core-shell cylinder (PCSC) and decoupling flexible cylinder (FCPR) models, and the structural parameters were estimated based on the fitting results of the scattering data. The stability of the coassembled AIP nanostructures in both fiber organization and bulk viscoelastic properties was also revealed via temperature-dependent SAXS analysis and oscillatory rheology measurements, respectively.

\section{INTRODUCTION}

Amyloid proteins consisting of a broad variety of amino acids has provided inspiration for developing functional materials due to their stability against mechanical, thermal, and chemical factors. ${ }^{1,2}$ These assemblies share the common structural property of a dense $\beta$-sheet organization, which enhances the stabilization of amyloid fibrils through multiple hydrogen bonding and noncovalent interactions. $^{3-6}$ In addition to their molecular organization, aggregation kinetics of these structures are interesting due to the aberrant deposition of their insoluble forms in natural organisms. This phenomenon is responsible for several classes of neurodegenerative disorders and is caused by the nucleationdependent formation of amyloid fibrils, which allows soluble monomers to assemble into existing filaments. ${ }^{7,8}$ Moreover, environmental conditions such as $\mathrm{pH}$, temperature, and ion concentrations have been demonstrated to alter the nucleation and growth kinetics of the amyloids in several studies. ${ }^{9,10}$ The hierarchical organization and thermal and mechanical stability exhibited by amyloid fibrils allow their use in the development of advanced functional materials for various applications. ${ }^{2,11-14}$ Previously, we showed that mixtures of oppositely charged amyloidinspired peptides (AIPs) form biocompatible self-assembled nanostructures and self-supporting gels with superior mechanical properties in the absence of external factors at physiological conditions. ${ }^{15}$ Although the material properties of these AIP assemblies have been analyzed at both nanometer and bulk scales using nanoindentation and oscillatory rheology techniques, respectively, coassembly kinetics of oppositely charged AIP-1 and AIP-2 peptides into supramolecular AIP nanostructures have not been investigated in detail, and the aggregation mechanisms involved in this process remain unknown.

Because of complex nature of the self-assembly process, it is quite difficult to monitor rapid assembly kinetics and to understand the parameters that play crucial roles in peptide aggregation and nanostructure formation under different conditions. Thioflavin $\mathrm{T}$ (ThT) assay is the most commonly used method for studying amyloid assembly. ${ }^{16}$ The binding of ThT to $\beta$-sheets results in red shift in excitation and emission spectra and strong increase in fluorescence intensity during the fibrillization process. ${ }^{17,18}$ The self-assembly of amyloid fibrils may also

Received: February 23, 2016

Revised: $\quad$ May 9, 2016

Published: June 6, 2016 
be observed directly through the fluorescence enhancement facilitated by the binding of ThT, which can be visualized by fluorescence imaging techniques. ${ }^{19,20}$ In addition to fluorescencebased approaches, small-angle X-ray scattering (SAXS) is a highly powerful technique for the mechanistic studies of peptide nanostructures, as the method allows the analysis of molecules at native conditions without manipulation of the sample, and may be utilized to perform time-resolved experiments using a high flux source. ${ }^{21}$ These techniques have been utilized for in-depth characterization of both functional and pathological amyloids $\mathrm{s}^{2,23}$ and facilitated the design of rapidly self-assembling, natureinspired synthetic peptide assemblies; however, greater insight into the organizational details and self-assembly characteristics of such synthetic peptide networks may further contribute to the development of functional designs for next-generation nanoscale materials.

In this study, we investigated the coassembly kinetics of oppositely charged AIPs by monitoring nanostructure formation and sol-gel transition at different peptide concentrations through various techniques, including ThT binding, real-time fluorescence imaging, turbidity assay, and in situ SAXS analysis. Coassembly kinetics were observed to depend strongly on initial peptide concentration, while peptide aggregation was found to be enhanced following fragmentation by sonication and exhibit amyloid-like sigmoidal assembly behavior. Structural properties of peptide assemblies were estimated by fitting the experimental scattering profiles of coassembled AIP nanostructures to theoretical models, and the mechanisms underlying their nanofibrous organization were analyzed with respect to the SAXS results. Mass fractal analysis of the AIP nanostructures and the conservation of their bulk material viscoelasticity at elevated temperatures also show the organizational stability of amyloid-inspired peptide architectures.

\section{EXPERIMENTAL SECTION}

Materials. All protected amino acids, Rink amide MBHA resin and 2-(1H-benzotriazol-1-yl)-1,1,3,3-tetramethyluronium hexafluorophosphate (HBTU) were purchased from NovaBiochem. Other chemicals used for peptide synthesis and material characterizations, including dichloromethane (DCM), dimethylformamide (DMF), acetonitrile, piperidine, acetic anhydride, $N, N$-diisopropylethylamine (DIAE), trifluoroacetic acid (TFA), thioflavin T (ThT), and uranyl acetate, were purchased from Fisher, Merck, Alfa Aesar, or Sigma-Aldrich. All chemicals and solvents used in this study were analytical grade.

Peptide Synthesis and Liquid Chromatography-Mass Spectrometry (LC-MS). Solid phase peptide synthesis method was used to synthesize AIP-1 (Ac-EFFAAE-Am) and AIP-2 (Ac-KFFAAK-Am) molecules. The details of the peptide synthesis and LC-MS analysis of the products were reported previously. ${ }^{15}$

Zeta Potential Measurements. Diluted AIP-1 and AIP-2 solutions at $0.04 \%(\mathrm{w} / \mathrm{v})$ concentration was prepared in water at $\mathrm{pH} 7$. AIP-1 and AIP-2 solutions were also mixed to obtain a coassembled nanofibrous architecture, incubated for $1 \mathrm{~h}$, and then diluted to a concentration of $0.04 \%(\mathrm{w} / \mathrm{v})$. Malvern Nano-ZS Zetasizer was used for the measurements.

ThT Binding Assay and Confocal Fluorescence Imaging. AIP-1 and AIP-2 solutions were prepared at 2, 1.75, 1.5, 1.25 and $1 \%(\mathrm{w} / \mathrm{v})$ concentration dissolving the peptide powders in double distilled water, separately. The $\mathrm{pH}$ of each solution was adjusted to 7.4 using $1 \mathrm{M}$ $\mathrm{NaOH}$. The same procedure was followed for all experiments. A Zeiss LSM 510 confocal microscope was used to monitor the coassembly process of AIPs through fluorescent ThT binding. 1:1 (v/v) mixtures of AIPs were prepared at different concentrations $(2,1.75,1.5,1.25$ and $1 \%(\mathrm{w} / \mathrm{v}))$ in water and at neutral $\mathrm{pH}$, and $100 \mu \mathrm{L}$ of each mixture was transferred to 96 -well plates. Immediately following the mixing of AIPs, a ThT solution in water was added onto the mixture at a final concentration of $216 \mu \mathrm{M}$. The samples were excited using a $458 \mathrm{~nm}$ Argon laser at $70 \%$ intensity and the emission was collected between 490 to $522 \mathrm{~nm}$ using appropriate filters to detect the binding of ThT to amyloid aggregates, which results in a shift in emission maxima from 440 to $490 \mathrm{~nm} .^{24}$ Fluorescence images were captured using a Zeiss EC Plan-Neofluar10 $\times$ objective at a resolution of $2048 \times 2048$ pixels. The dimensions of the scanned volume within the AIP mixtures were $1272.17 \mu \mathrm{m} \times 1272.17 \mu \mathrm{m} \times 25 \mu \mathrm{m}$. The $Z$-depth separation between the planes was $5 \mu \mathrm{m}$. The pixel dwell time, master gain, digital gain, digital offset, and pinhole radius values were $0.80 \mu \mathrm{s}, 668,1.09,-0.05$, and $336 \mu \mathrm{m}$, respectively. Fluorescence intensity changes were quantified with respect to time in the same defined region of the interest (ROIs) for $1 \mathrm{~h}$ at $25{ }^{\circ} \mathrm{C}$ for all groups. The time interval between each fluorescent measurement and image capture was $2 \mathrm{~min}$. Three-dimensional (3D) image construction of each time interval was performed using Zeiss LSM 510 software, and the ImageJ program was used for the $3 \mathrm{D}$ video construction of the images at a rate of 1 frame per second. Results were reported as the average of three repeats and all experiments were conducted at the same sample positioning and microscope configurations.

Turbidity Assay. On hundred microliters of 1:1 (v/v) mixtures of AIPs were prepared as described above and transferred to 96 well plates. Turbidity of AIP mixtures were then monitored as optical density at $313 \mathrm{~nm}$ on a SpectraMax M5Microplate Reader for $1 \mathrm{~h}$ at $25^{\circ} \mathrm{C}$. Measurements were reported as the average of three repeats. For the analysis of the effects of fragmentation on turbidity kinetics, AIP mixtures were sonicated for different time periods $(1,2.5$, or $5 \mathrm{~min})$ using an ultrasonic bath (VWR, USC100T, $230 \mathrm{~V}, 60 \mathrm{~Hz}$ ) prior to turbidity measurements. Following sonication, samples were immediately transferred to 96-well plates and analyzed under identical conditions as the nonsonicated AIP mixtures.

SAXS Measurements and Data Fitting. SAXS measurements were performed using the SAXS/WAXS beamline 23A of the National Synchrotron Radiation Research Center (NSRRC), Hsinchu, Taiwan. The photon energy and sample-to-detector distance were set to be $15 \mathrm{keV}$ and $3312.99 \mathrm{~mm}$, respectively, in order to cover a scattering vector $\mathbf{q}=(4 \pi / \lambda) \sin (\theta / 2)$ from 0.006 to $0.40 \AA^{-1}$ (where $\theta$ is the scattering angle and $\lambda$ is the wavelength of the incident $\mathrm{X}$-rays). Scattered X-rays were collected by means of a $2 \mathrm{D}$ CCD detector. The collected $2 \mathrm{D}$ data were circularly averaged to give a $1 \mathrm{D}$ scattering intensity distribution as a function of the scattering vector, treated with background subtraction, and transmission correction and normalized to absolute scattering intensity. For concentration-dependent SAXS experiments, equal volumes of $2,1.75$, and $1 \%(\mathrm{w} / \mathrm{v})$ AIP-1 were mixed with $2,1.75$, and $1 \%(\mathrm{w} / \mathrm{v})$ AIP-2, respectively, and AIP mixtures were immediately loaded in $2.5 \mathrm{~mm}$-thick cells with Kaptonwalled windows at a constant temperature of $25{ }^{\circ} \mathrm{C}$. Samples were incubated for $1 \mathrm{~h}$ before the measurements and subsequently had their scattering profiles collected. For the in situ SAXS analysis of $2 \%(\mathrm{w} / \mathrm{v})$ AIPs, the time of the mixing was accepted as zero and SAXS measurements were conducted at defined time intervals ( $30 \mathrm{~s} \leq t \leq 300 \mathrm{~s}, \Delta t=$ $30 \mathrm{~s}$, and at $1 \mathrm{~h}$ ). In addition to time-dependent analysis, the effect of the temperature on $2 \%(\mathrm{w} / \mathrm{v})$ AIPs was also analyzed through SAXS measurements at different temperatures $\left(25{ }^{\circ} \mathrm{C} \leq T \leq 64{ }^{\circ} \mathrm{C}\right.$, $\Delta T=3{ }^{\circ} \mathrm{C}$ ) following $1 \mathrm{~h}$ of incubation. Scattering profiles of the AIP nanostructures were fitted into a combination of the polydisperse core-shell cylinder (poly core-shell cylinder, PCSC) ${ }^{25}$ and decoupling flexible cylinder (Flexible Cylinder Poly Radius: FCPR) ${ }^{26,27}$ models, which estimate the scattering properties of a polydisperse, right circular cylinder with a core-shell scattering length density profile and a cylinder in a flexible, ordered fractal aggregation form, respectively (Figure S6). The shell thickness on the flat ends of the cylinder is independent of the shell thickness on the radial surface. The polydispersity of the cylinder core radius was modeled using a lognormal distribution. The overall intensity was obtained by calculating the scattering from each particle size present and weighting it by the normalized distribution. The details of the models and fitting procedures are provided in the Supporting Information (in the SAXS data fitting and modeling section). 
Imaging of the Coassembled Supramolecular AIP Nanofibers and Their Gels. For TEM analysis, 2\% (w/v) AIP-1 and AIP-2 solutions were mixed to obtain coassembled nanofibrous systems and incubated for $15 \mathrm{~min}$ or $1 \mathrm{~h}$. TEM sample preparation for coassembled AIP nanofibers were carried out according to the previous reported protocol. ${ }^{15}$ For SEM imaging of coassembled supramolecular AIP gels, AIP-1 and AIP-2 solutions were mixed at a 1:1 volume ratio at different concentrations $(2,1.75,1.5,1.25$, and $1 \%(\mathrm{w} / \mathrm{v}))$ in water and at neutral $\mathrm{pH}$ and kept at room temperature for sol-gel transition on a Si wafer. Complete gelation was observed within $1 \mathrm{~h}$ for the AIP mixtures prepared at $2,1.75$, and $1.5 \%(\mathrm{w} / \mathrm{v})$ concentrations, and SEM imaging using a FEI Quanta 200 FEG scanning electron microscope equipped with an ETD detector was performed on these samples after the critical point drying of the samples. ${ }^{15}$ AFM samples of the coassembled AIP nanofibers was prepared as described for TEM imaging, incubated for $1 \mathrm{~h}$, diluted to a concentration of $0.05 \%(\mathrm{w} / \mathrm{v})$, and drop casted onto a Si wafer for drying at room temperature. AFM measurements were then performed in noncontact mode using a commercial microscope (MFP3D, Asylum Research).

Oscillatory Rheology. Temperature-dependent rheology measurements were performed in the range of $25-60{ }^{\circ} \mathrm{C}$ on $2 \%(\mathrm{w} / \mathrm{v})$ AIP mixtures, which were prepared as described for TEM and AFM measurements and incubated for $1 \mathrm{~h}$ to complete sol-gel transition prior to analysis. The total volume of the sample was $250 \mu \mathrm{L}$, and a PP25-SN17979 measuring device with a diameter of $25 \mathrm{~mm}$ was used for rheology measurements. The measuring distance was determined as $0.5 \mathrm{~mm}$. Time sweep analysis was carried out at elevated temperatures under $10 \mathrm{rad} / \mathrm{s}$ angular frequency and $0.1 \%$ strain magnitude. The heating rate of the sample was $10^{\circ} \mathrm{C} \mathrm{min}-1$ with linear ramping. An Anton Paar MCR-301 Rheometer was used for the analysis. A solvent trap included with the instrument was used to maintain a humid environment and prevent the drying of the sample during measurement. Measurements are reported as average of the three repeats.

\section{RESULTS AND DISCUSSION}

Noncovalent interactions between short peptide molecules serve as a driving force for formation of coassembled nanostructures under physiological conditions ${ }^{28}$ and facilitate the growth of supramolecular fibrillar structures. ${ }^{29,30}$ In particular, positively charged amino acids can interact with negatively charged amino acids between peptide molecules, thereby playing an important role in mediating the peptide self-assembly process. ${ }^{31,32}$ AIP-1 and AIP-2 molecules consist of two Glu and Lys residues, which carry -2 and +2 net charges at around $\mathrm{pH} 7$ in water (Figure S1). The zeta potential measurements of the individual AIP-1 and AIP-2 solutions were also pointed their negative and positive charges in this condition (Figure S2). Upon mixing peptide solutions with 1:1 molar ratio, the overall charge neutralization triggered coassembly of AIPs into supramolecular nanostructures and three-dimensional networks (Figure S2 and S3). Hydrophobic-FFAA-domain of AIPs also enhanced repetitive H-bonding, hydrophobic, and aromatic interactions, which are commonly present in native amyloid aggregation. ${ }^{33-35}$

To study the coassembly kinetics of AIP molecules at physiological conditions, AIP-1 and AIP-2 solutions were prepared at varying concentrations between $1 \%$ and $2 \%(\mathrm{w} / \mathrm{v})$, and mixed at a molar ratio of $1: 1$ at the physiological conditions. Coassembly kinetics of the AIPs were monitored via addition of ThT, a small molecule, ${ }^{36,37}$ which binds the $\beta$-sheets of the AIP aggregates and exhibits an increase in fluorescence emission intensity during coassembly (Figure 1). Time-dependent increase in fluorescence intensities were monitored using confocal fluorescent microscopy and normalized to maximum ThT intensity to analyze the peptide assembly process (Figure $1 \mathrm{~b}$ and 2). This technique facilitates the analysis of turbid peptide solutions due
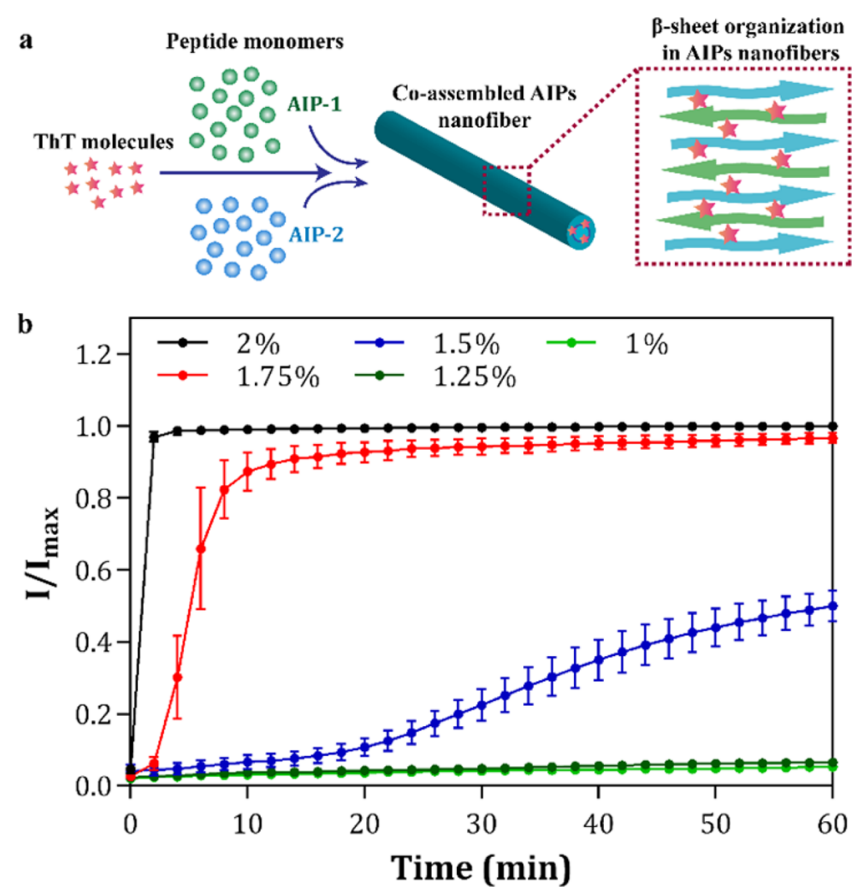

Figure 1. (a) A small fluorescent dye molecule, thioflavin $T$ (ThT), is used as a probe for monitoring the coassembly of AIP-1 and AIP-2 molecules into nanostructures. During the coassembly process, ThT molecules coordinate along the surface side-chain grooves running parallel to the long axis of the $\beta$-sheets. This organization of the ThT molecules within the coassembled AIP nanostructures lead to an increase in their fluorescence intensity, and the kinetics of coassembly process can thereby be monitored. (b) The normalized fluorescence intensity changes during the coassembly process of the AIPs prepared at different peptide concentrations.

to the formation of opaque supramolecular nanostructure networks. In addition, $z$-stacking within the AIP mixtures allowed tracking the changes in $3 \mathrm{D}$ fluorescence intensity and correlates this information to the formation of aggregates during the coassembly process (Supplementary Videos V1-V5).

AIPs showed different coassembly kinetics depending on the peptide concentration (Figure $1 \mathrm{~b})$. Above $1.5 \%(\mathrm{w} / \mathrm{v})$ concentration, the AIP mixtures rapidly self-assemble into supramolecular systems within $1 \mathrm{~h}$. On the other hand, the coassembly process slowed down and did not reach to maximum fluorescence within $1 \mathrm{~h}$ for $1.25 \%$ and $1 \%(\mathrm{w} / \mathrm{v})$ AIP samples. Although an initial delay of up to $20 \mathrm{~min}$ was observed in the aggregation of AIPs at the threshold concentration of $1.5 \%(\mathrm{w} / \mathrm{v})$, this delay was followed by a rapid increase in selfassembly, which also failed to reach the rates observed in higher AIP concentrations after $1 \mathrm{~h}$. In the literature, ThT binding assay has been used to determine the kinetics of the aggregation process and important parameters that effect the aggregation behavior of amyloids. It is known that higher initial concentrations increase the assembly kinetics and decrease the lag-time of the aggregation process in amyloid solutions. ${ }^{38-40}$ In addition, growth curves associated with amyloid formation typically approximate a sigmoidal shape, in which the nucleation of the monomers is followed by their rapid aggregation into filamentous nanostructures. ${ }^{41}$ AIP coassembly kinetics were overall similar to these displayed by native amyloid assemblies, as the peptide molecules were observed to form self-supporting three-dimensional networks (Figure S3) under sigmoidal-like formation kinetics over a concentration of $1.25 \%$. Although 


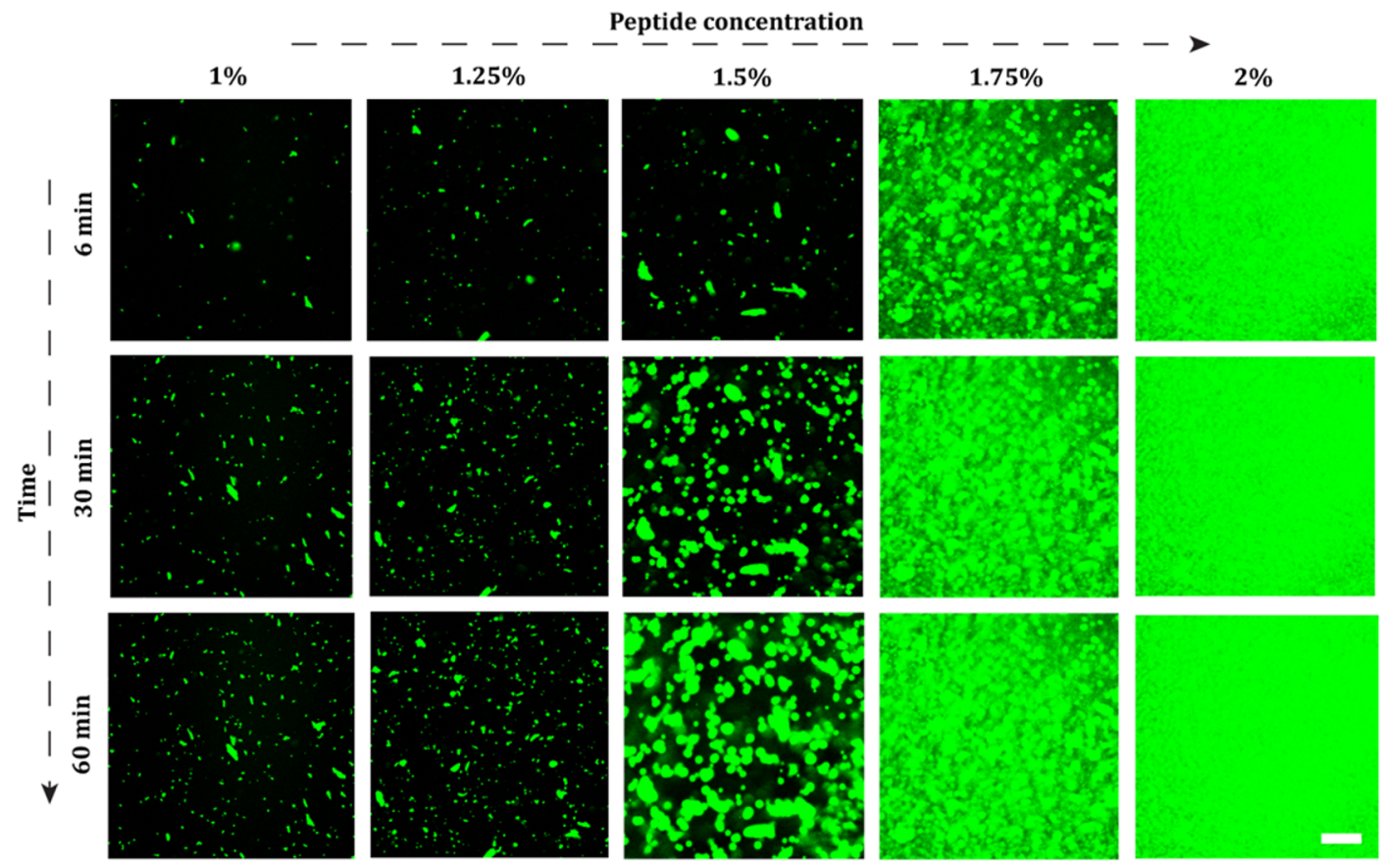

Figure 2. Time- and concentration-dependent coassembly of oppositely charged AIPs at physiological conditions into supramolecular nanostructures. The aggregation kinetics of the AIPs were visualized using in situ confocal microscopy imaging during the coassembly process at different concentrations (all image scale bars are $200 \mu \mathrm{m}$ ).

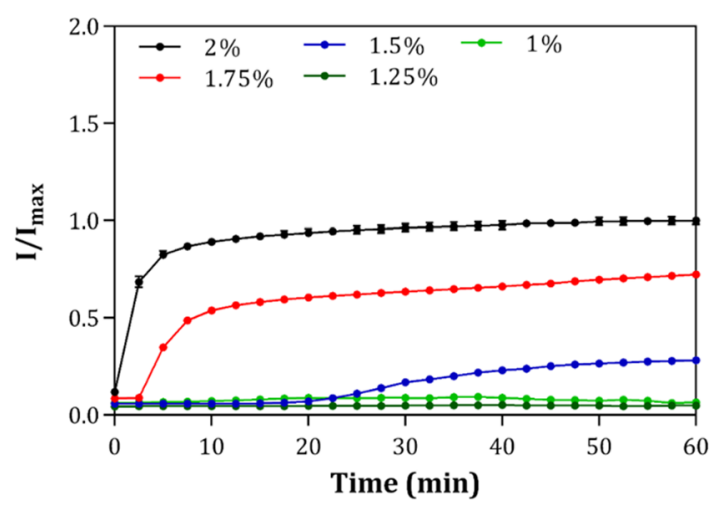

Figure 3. Turbidity of AIP solutions at different concentrations was monitored as optical density at $313 \mathrm{~nm}$ under physiological conditions. Increase in the turbidity of the solutions correlate with nanostructure formation and sol-gel transition. AIP-1 and AIP-2 molecules rapidly self-assemble into the nanostructures a concentration over $1.25 \%(\mathrm{w} / \mathrm{v})$ concentration within $1 \mathrm{~h}$.

initial nucleated aggregates have been detected via ThT binding for all concentrations of AIP mixtures (Figure 2 and Supplementary Videos V1-V5), their coassembly kinetics reach to maxima within $1 \mathrm{~h}$ only for concentrations above $1.5 \%(\mathrm{w} / \mathrm{v})$.

Turbidity assay was also used to follow the self-assembly process and analyze peptide aggregation kinetics ${ }^{42,43}$ in addition to the ThT binding assay. AIP mixtures were prepared as described for the ThT binding assay and their turbidities at $313 \mathrm{~nm}$ were normalized to their maximum absorbance to determine the time-dependent assembly of the peptide networks (Figure 3). Turbidity kinetics of the AIPs showed a similar pattern with the ThT binding kinetics, exhibiting a time-dependent increase within $1 \mathrm{~h}$ at concentrations over $1.25 \%(\mathrm{w} / \mathrm{v})$. A rapid increase was also observed in the turbidity of the $1.5 \%(\mathrm{w} / \mathrm{v})$ sample after $20 \mathrm{~min}$, supporting our ThT results. Although two different assays have been utilized for analyzing the behavior of AIP mixtures, both ThT binding and turbidity assays overall suggest that the AIPs exhibit amyloid-like aggregation and sigmoidal assembly kinetics above a concentration of $1.25 \%(\mathrm{w} / \mathrm{v})$. As a control experiment, turbidities of AIP-1 and AIP-2 solutions were also measured separately at $313 \mathrm{~nm}$ and normalized to the maximum absorbance of the curves; however, no increase in solution turbidity could be detected when the peptide components were not mixed with one another (Figure S4).

Fragmentation of amyloid aggregates may accelerate the assembly kinetics of fibril formation, since amyloid fibril ends behave as nucleation point for fiber elongation. The analogy between enzymatic reactions and amyloid aggregation has previously been underlined in the literature. ${ }^{39}$ Sonication is a way to break the nucleated amyloid aggregates via sound waves, and the technique was used to enhance the spontaneous fibril formation of amyloid peptides. ${ }^{44}$ In this study, sonication was applied for different time periods after $1 \%(\mathrm{w} / \mathrm{v})$ AIP-1 and AIP-2 solutions were mixed at physiological conditions, and the turbidity changes exhibited by AIP mixtures were then monitored at $313 \mathrm{~nm}$ for $1 \mathrm{~h}$ (Figure S5). Although 1\% (w/v) AIPs solution was not observed to form three-dimensional networks within $1 \mathrm{~h}$ in both ThT binding and turbidity assays; turbid AIP mixtures were obtained at this concentration when the samples were sonicated (Figure S5b). In addition, short-term sonication of $1 \%(\mathrm{w} / \mathrm{v})$ AIPs before the turbidity study enhanced the coassembly process, and no change was observed on the turbidity of the solutions within $1 \mathrm{~h}$ (Figure S5b). In addition to the effects of monomer concentration on coassembly kinetics, fragmentation via sonication also enhanced the formation of supramolecular AIP nanostructures at physiological conditions, which is in agreement with the previous records on amyloid aggregation behavior.

To characterize structural properties of the peptide nanostructures, AIP mixtures were incubated for $1 \mathrm{~h}$ under physiological 
conditions in a $2.5 \mathrm{~mm}$ thick, Kapton-walled sample cell and analyzed by SAXS to determine their scattering profiles. Scattering intensity $(I)$ was recorded as a function of the magnitudes of

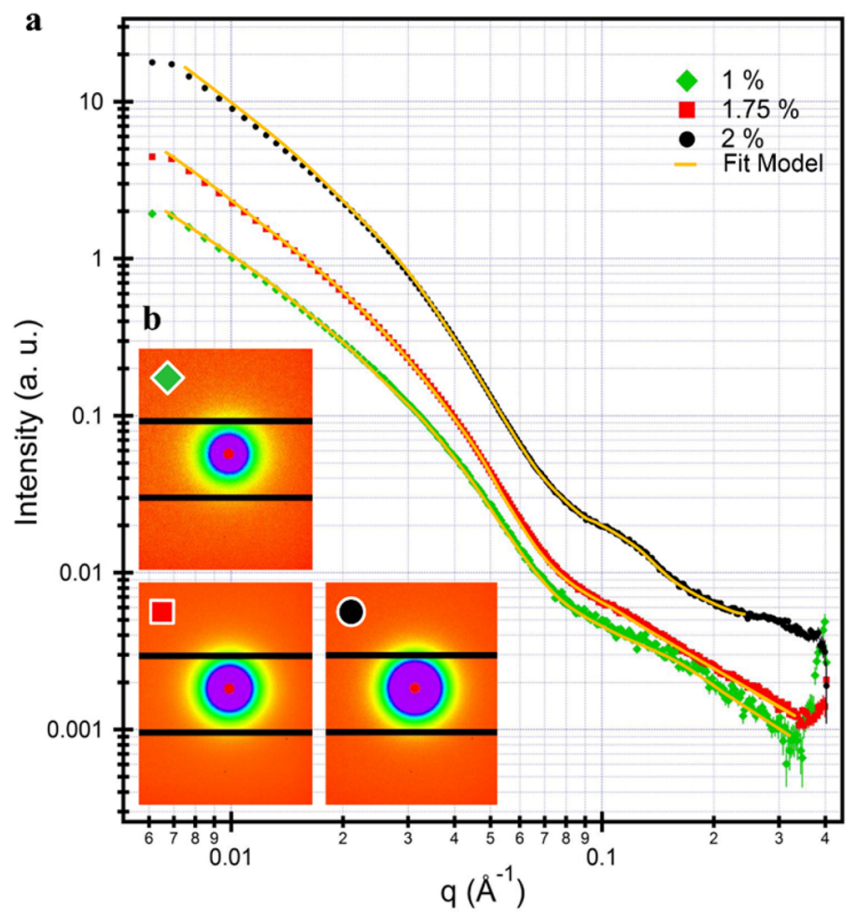

Figure 4. (a) SAXS profiles of coassembled $2 \%, 1.75 \%$, and $1 \%(\mathrm{w} / \mathrm{v})$ AIP nanostructures after $1 \mathrm{~h}$. Changes in scattering intensity are given as a function of the scattering wave vectors $\mathbf{q}\left(\AA^{-1}\right)$, and the data were best-fitted to a combination of PCSC and FCPR models. (b) The 2D patterns of coassembled AIPs nanostructures at different concentrations in water. scattering vectors $\mathbf{q}\left(\AA^{-1}\right)$ of the coassembled AIPs at different concentrations (Figure 4), and scattering data in the Guinier region was used to determine the radius of gyration for the coassembled AIP nanostructures (Figure 4). Data fitting and processing were performed by using IGOR Pro $6.3,{ }^{45}$ and the data were best-fitted to a combined model defined by the polydisperse core-shell cylinder (PCSC) ${ }^{25}$ and decoupling flexible cylinder (FCPR) ${ }^{26,27}$ models (Figures 5a and S6). The obtained structural parameters are shown in Table 1 . The electron density of core section was higher than the shell, possibly due to the tendency of the hydrophobic domains of AIPs (-FFAA-) to stay in the inner region of coassembled AIP nanofibers (Figure 5b). In addition, the shell region consisting of hydrophilic -Lys and -Glu residues, provided a contact area with water molecules. The electron densities were also found to change repetitively at a periodicity of $3.1 \mathrm{~nm}$ across the longitudinal axis of the fiber. $\pi-\pi$ stacking of aromatic residues and twisted $\beta$-sheet organization of AIPs in the fibers may cause this periodicity exhibited by electron density. The nanofibrous organization of the coassembled AIP supramolecular nanostructures were also observed using AFM imaging of peptide networks dried on Si wafers (Figure 5c) and are consistent with the model developed in the light of the scattering data.

In situ SAXS measurements were also performed on a $2 \%(\mathrm{w} / \mathrm{v})$ AIP mixture to monitor its structural organization during the coassembly process. Experiments were performed at this concentration due to the rapid coassembly kinetics of the $2 \%(\mathrm{w} / \mathrm{v})$ AIP mixture, which would allow the observation of both short- and long-term changes in the peptide network. The first scattering profile was collected immediately after $(t \sim 30 \mathrm{~s})$ the mixing and transfer of AIPs into capillary tubes, while following measurements were acquired every $30 \mathrm{~s}$ within the first $5 \mathrm{~min}$. Scattering profiles were then analyzed to determine whether a structural or organizational transition had occurred
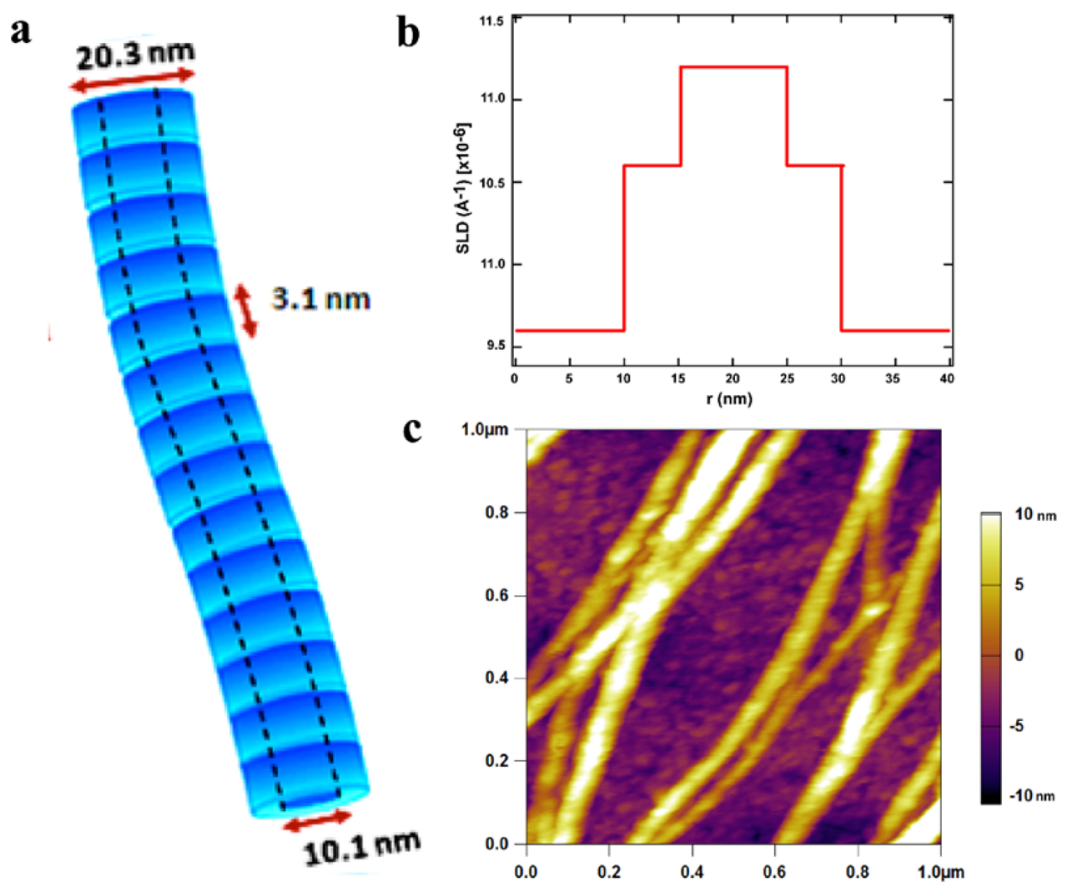

Figure 5. $(\mathrm{a}, \mathrm{b})$ The proposed structural model for coassembled AIP nanostructures. The electron density of the core part is higher than the shell, as the hydrophobic domain of the AIPs (-FFAA-) prefers to remain in the inner part of the coassembled AIP nanofibers. In the perpendicular fiber axis, the electron densities also change at a periodicity of $3.1 \mathrm{~nm} . \pi-\pi$ stacking of aromatic residues and twisted $\beta$-sheet organization of AIPs in the fibers may cause this periodicity exhibited by electron density. (c) AFM image of the coassembled AIP nanofibers and their bundles dried on a $S i$ wafer. 
Table 1. Fitting Results of SAXS Data of Coassembled AIP Nanostructures at Different Concentrations in Water at Neutral pH

\begin{tabular}{|c|c|c|c|c|}
\hline model & parameters & $1 \%$ & $1.75 \%$ & $2 \%$ \\
\hline \multirow[t]{10}{*}{ PCSC } & $\mathrm{R}_{\mathrm{p}}$, core radius $(\AA)$ & $47.6 \pm 0.60$ & $51.6 \pm 0.80$ & $48.7 \pm 0.70$ \\
\hline & Radial shell thickness $(\AA)$ & $55.7 \pm 0.50$ & $53.5 \pm 0.90$ & $52.3 \pm 0.70$ \\
\hline & Radial polydispersity, sigma & 0.9 & 0.8 & 0.6 \\
\hline & $H_{\mathrm{p}}$, core length $(\AA)$ & $23.9 \pm 0.10$ & $24.0 \pm 0.10$ & $21.6 \pm 0.10$ \\
\hline & face shell thickness $(\AA)$ & $3.2 \pm 0.10$ & $3.1 \pm 0.10$ & $4.5 \pm 0.10$ \\
\hline & $R_{\mathrm{l}}$, shell radius $(\AA)$ & $103.3 \pm 0.80$ & $105.1 \pm 0.90$ & $101.2 \pm 0.50$ \\
\hline & $H_{1}$, shell length $(\AA)$ & $30.3 \pm 0.10$ & $30.2 \pm 0.10$ & $30.6 \pm 0.10$ \\
\hline & SLD core $\left(\AA^{-2}\right)$ & $10.8 \times 10^{-6}$ & $11.1 \times 10^{-6}$ & $11.3 \times 10^{-6}$ \\
\hline & SLD shell $\left(\AA^{-2}\right)$ & $10.1 \times 10^{-6}$ & $10.2 \times 10^{-6}$ & $10.6 \times 10^{-6}$ \\
\hline & SLD solvent $\left(\AA^{-2}\right)$ & $9.6 \times 10^{-6}$ & $9.7 \times 10^{-6}$ & $9.6 \times 10^{-6}$ \\
\hline \multirow[t]{6}{*}{ FCPR } & $L$, contour length $(\AA)$ & $420.2 \pm 0.90$ & $430.1 \pm 0.30$ & $434.7 \pm 0.40$ \\
\hline & b, Kuhn length $(\AA)$ & $60.6 \pm 0.10$ & $60.5 \pm 0.10$ & $61.3 \pm 0.20$ \\
\hline & $R$, radius $(\AA)$ & $103.3 \pm 0.80$ & $105.1 \pm 0.90$ & $101.2 \pm 0.70$ \\
\hline & polydispersity of radius & 3.2 & 5.6 & 4.3 \\
\hline & SLD cylinder $\left(\AA^{-2}\right)$ & $10.2 \times 10^{-6}$ & $11.6 \times 10^{-6}$ & $11.9 \times 10^{-6}$ \\
\hline & SLD solvent $\left(\AA^{-2}\right)$ & $9.7 \times 10^{-6}$ & $9.5 \times 10^{-6}$ & $9.6 \times 10^{-6}$ \\
\hline
\end{tabular}

${ }^{a}$ Core radius $=R_{\mathrm{p}}$, core length $=H_{\mathrm{p}}$ (the mean core radius is $R_{\mathrm{o}}$ ); the shell radius and shell length incorporate the dimensions of the bare particle ( $H_{1}=H_{\mathrm{p}}+2 \times$ face thickness and $R_{1}=R_{\mathrm{p}}+$ radial thickness). Sigma is equivalent to the standard deviation of the log-normal distribution.
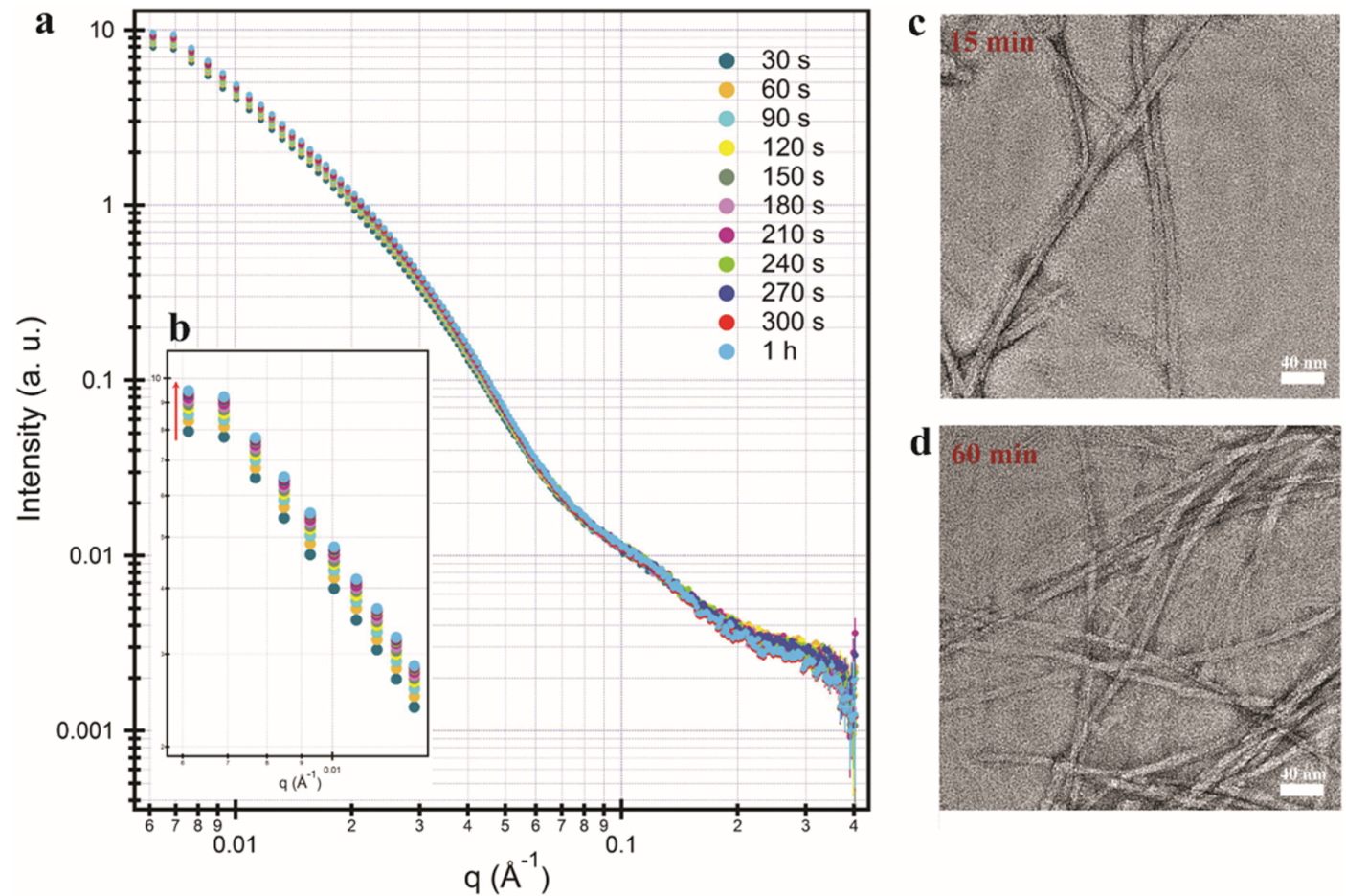

Figure 6. (a) In situ SAXS measurements of $2 \%$ AIPs in water at neutral $\mathrm{pH}$ during coassembly. The scattering intensity collected at the low-q region increased during the coassembly due to the nanofiber formation (b). TEM images of the nanofibers prepared from the dilutions of coassembled $2 \%$ AIPs for 15 (c) and $60 \mathrm{~min}(\mathrm{~d})$.

during the coassembly process (Figure 6a, b). All scattering profiles collected within the first $5 \mathrm{~min}$ were best-fitted to the same structural model given in Figure S6, and the results showed that AIP-1 and AIP-2, when mixed above the critical sol-gel transition concentration at $\mathrm{pH} 7$ in water, are able to coassemble into nanofibers without any transition from another structural organization within the collected time intervals. In addition, no structural or organizational changes were observed on the TEM images of the AIP nanostructures prepared from the dilutions of $2 \%$ coassembled AIPs for $15 \mathrm{~min}$ and $1 \mathrm{~h}$, further supporting our SAXS results (Figure 6c,d).
Temperature-dependent structural stability of coassembled $2 \%(\mathrm{w} / \mathrm{v})$ AIP nanostructures for $1 \mathrm{~h}$ was also investigated by SAXS analysis performed at elevated temperatures between 25 to $64{ }^{\circ} \mathrm{C}$ (Figure $7 \mathrm{a}$ ). In addition to the scattering profiles analysis of peptide coassemblies at different temperatures, which were best fitted to the flexible core-shell cylinder model described above, fractal mass analysis was performed to probe the AIP nanostructures in high $\mathbf{q}$ regimes (Figure $7 \mathrm{~b}$ ). The scattering exponent $\alpha$ can be estimated from the slope of a $\log I(\mathbf{q})$ versus $\log \mathbf{q}$ curve derived from scattering data. ${ }^{46}$ In a high regime, $\alpha$ is equal to the mass fractal dimension, $D_{\mathrm{m}}$, revealing the degree of compactness of the scattering object. ${ }^{21,46}$ 


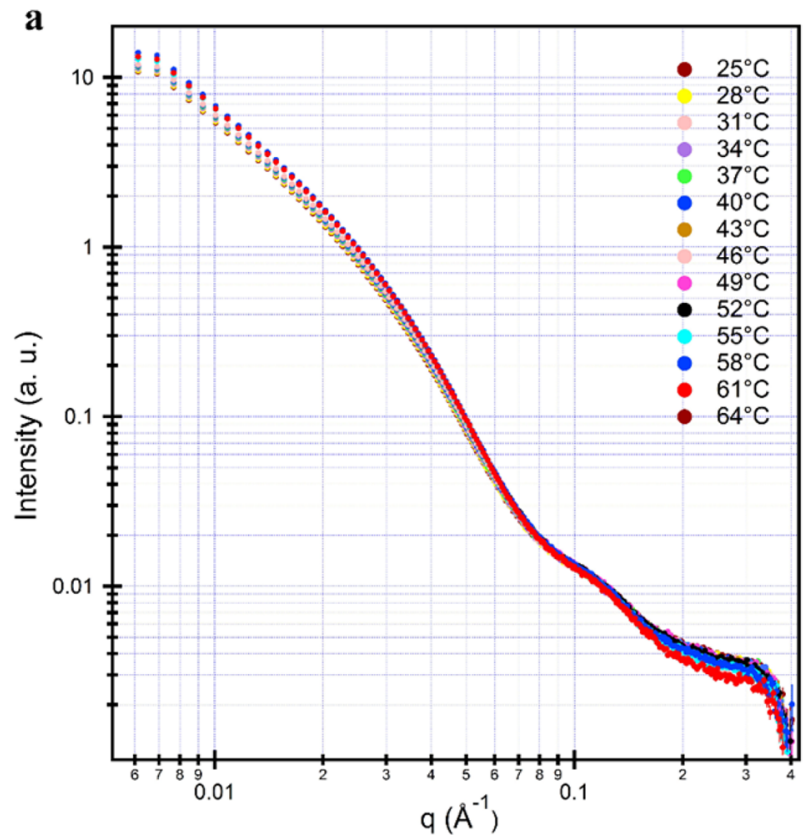

\begin{tabular}{|c|c|c|}
\hline & $\begin{array}{c}\text { Temperature } \\
\left({ }^{\circ} \mathrm{C}\right)\end{array}$ & $\begin{array}{c}\mathrm{D}_{\mathrm{m}} \text { (Fractal } \\
\text { mass) }\end{array}$ \\
\hline \multirow{13}{*}{$2 \%$} & 25 & 1.89 \\
\hline & 28 & 1.88 \\
\hline & 31 & 1.87 \\
\hline & 34 & 1.87 \\
\hline & 37 & 1.87 \\
\hline & 40 & 1.86 \\
\hline & 43 & 1.87 \\
\hline & 46 & 1.86 \\
\hline & 52 & 1.86 \\
\hline & 55 & 1.86 \\
\hline & 58 & 1.86 \\
\hline & 61 & 1.82 \\
\hline & 64 & 1.82 \\
\hline
\end{tabular}

Figure 7. (a) SAXS profiles of $2 \%$ coassembled AIP nanostructures at different temperatures. (b) Increase in temperature did not disturb the organization of the AIP nanofibers and the density of the nanofibers slightly changed for elevated temperatures. Fractal dimension values indicate 3D mass fractals $\left(1<D_{\mathrm{m}}<3\right)$ and also support the PCSC model as a mass fractal.

For semiordered structures, $D_{\mathrm{m}}$ has a value between 1 and $3,^{47}$ and higher $D_{\mathrm{m}}$ values indicate the denser structural organization of the coassembled peptide aggregates. ${ }^{31}$ Hence, it was expected to obtain loose nanostructures and lower $D_{\mathrm{m}}$ values at higher temperatures, which weaken noncovalent interactions between the AIP molecules. However, $D_{\mathrm{m}}$ values of $2 \%(\mathrm{w} / \mathrm{v})$ coassembled AIP nanostructures changed only slightly up to $61{ }^{\circ} \mathrm{C}$, and a rapid decrease was observed above this temperature (Figure $7 \mathrm{~b}$ ). Bulk viscoelastic character of $2 \%(\mathrm{w} / \mathrm{v})$ coassembled AIP nanostructure network was also analyzed at elevated temperature range for $1 \mathrm{~h}$ to confirm out SAXS experiments (Figure 8),

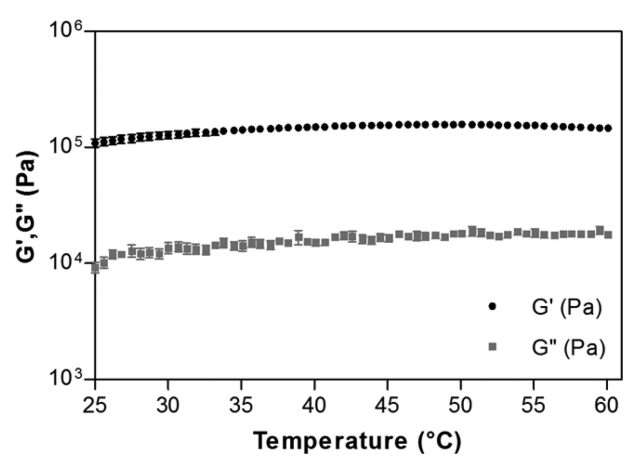

Figure 8. Effect of temperature on the mechanical stability of coassembled $2 \%(\mathrm{w} / \mathrm{v})$ supramolecular AIP nanostructure network for $1 \mathrm{~h}$. The coassembled network preserved viscoelastic behavior at elevated temperatures.

and the viscoelastic gel properties of the coassembled AIPs were found to be preserved in the $25-64{ }^{\circ} \mathrm{C}$ range, which agrees with the nonsignificant change in structural organization monitored via SAXS analysis in this temperature interval.

\section{CONCLUSION}

The design of short self-assembling synthetic peptides, inspired by noncovalent interactions in native amyloid aggregations, allows the formation of coassembled supramolecular nanostructures at neutral $\mathrm{pH}$ through hydrogen bonding, hydrophobic, and electrostatic forces. The interactions between the oppositely charged short amyloid-inspired AIP-1 and AIP-2 peptide molecules supported the formation of stable nanofibers at physiological conditions. The coassembly kinetics of the peptides displayed nucleation-dependent, amyloid-like aggregation characteristics above a critical monomer concentration, which was monitored via both ThT binding and turbidity assays. In addition, fragmentation of the AIP nanostructures by ultrasonication enhanced the aggregation of the $1 \%(\mathrm{w} / \mathrm{v})$ AIP mixture, which normally could not reach maximum turbidity within $1 \mathrm{~h}$. Models developed by the detailed SAXS analysis of coassembled AIP nanostructures also revealed the core-shell nanofibrous organization of the oppositely charged short AIP molecules, which is likely to stem from the hydrophobicity of aromatic residues and hydrophilicity of -Lys and -Glu residues on AIP-1 and AIP-2 molecules, respectively. In addition to the structural model and estimated organizational parameters, in situ SAXS analysis suggests that no transition in the structural organization of peptide molecules during the coassembly process of $2 \%(\mathrm{w} / \mathrm{v})$ AIP mixture. The stability of the coassembled AIP nanostructures in both nanoscale fiber organization and bulk viscoelastic properties was shown via temperature dependent SAXS analysis and oscillatory rheology measurements. Although a variety of short peptide designs and self-assembled architectures were reported to display amyloid-like structural organizations in the literature, more information is required for their in-depth structural characterization. In this study, we systematically investigated the in situ amyloid-like aggregation kinetics of two oppositely charged short peptide molecules into supramolecular nanostructures. More information about the selfassembly process of short synthetic peptides can facilitate the development of therapeutic strategies for protein-folding disorders and the design of improved materials derived from self-assembling amyloid inspired peptides. 


\section{ASSOCIATED CONTENT}

\section{S Supporting Information}

The Supporting Information is available free of charge on the ACS Publications website at DOI: 10.1021/acs.langmuir.6b00704.

LC-MS results; SEM images of coassembled AIP nanofibers; the supplementary results of time and sonication dependent turbidity experiments; and the details of SAXS data fitting and modeling.(PDF)

Confocal microscopy videos.(ZIP)

\section{AUTHOR INFORMATION}

\section{Corresponding Author}

*E-mail: moguler@unam.bilkent.edu.tr. Fax: +90 (312) 266 4365. Telephone: +90 (312) 2903552.

Notes

The authors declare no competing financial interest.

\section{ACKNOWLEDGMENTS}

This work is partially supported by grants TUBITAK (109T603), TUBA-GEBIP, and FP7Marie Curie IRG. G.C. is supported by TUBITAK-BIDEB 2211-C Ph.D. fellowship. We thank M. Guler for help in TEM imaging.

\section{REFERENCES}

(1) VandenAkker, C. C.; Engel, M. F.; Velikov, K. P.; Bonn, M.; Koenderink, G. H. Morphology and persistence length of amyloid fibrils are correlated to peptide molecular structure. J. Am. Chem. Soc. 2011, 133 (45), 18030-18033.

(2) Knowles, T. P.; Buehler, M. J. Nanomechanics of functional and pathological amyloid materials. Nat. Nanotechnol. 2011, 6 (8), 469479.

(3) Smith, J. F.; Knowles, T. P.; Dobson, C. M.; MacPhee, C. E.; Welland, M. E. Characterization of the nanoscale properties of individual amyloid fibrils. Proc. Natl. Acad. Sci. U. S. A. 2006, 103 (43), 15806-15811.

(4) Sawaya, M. R.; Sambashivan, S.; Nelson, R.; Ivanova, M. I.; Sievers, S. A.; Apostol, M. I.; Thompson, M. J.; Balbirnie, M.; Wiltzius, J. J.; McFarlane, H. T. Atomic structures of amyloid cross- $\beta$ spines reveal varied steric zippers. Nature 2007, 447 (7143), 453-457.

(5) Nelson, R.; Sawaya, M. R.; Balbirnie, M.; Madsen, A. Ø.; Riekel, C.; Grothe, R.; Eisenberg, D. Structure of the cross- $\beta$ spine of amyloidlike fibrils. Nature 2005, 435 (7043), 773-778.

(6) Morris, K. L.; Rodger, A.; Hicks, M. R.; Debulpaep, M.; Schymkowitz, J.; Rousseau, F.; Serpell, L. C. Exploring the sequencestructure relationship for amyloid peptides. Biochem. J. 2013, 450 (2), 275-283.

(7) Anthony, N. R.; Mehta, A. K.; Lynn, D. G.; Berland, K. M. Mapping amyloid- $\beta(16-22)$ nucleation pathways using fluorescence lifetime imaging microscopy. Soft Matter 2014, 10 (23), 4162-4172.

(8) Knowles, T. P.; Waudby, C. A.; Devlin, G. L.; Cohen, S. I.; Aguzzi, A.; Vendruscolo, M.; Terentjev, E. M.; Welland, M. E.; Dobson, C. M. An analytical solution to the kinetics of breakable filament assembly. Science 2009, 326 (5959), 1533-1537.

(9) Buell, A. K.; Galvagnion, C.; Gaspar, R.; Sparr, E.; Vendruscolo, M.; Knowles, T. P.; Linse, S.; Dobson, C. M. Solution conditions determine the relative importance of nucleation and growth processes in $\alpha$-synuclein aggregation. Proc. Natl. Acad. Sci. U. S. A. 2014, 111 (21), 7671-7676.

(10) Scheibel, T.; Bloom, J.; Lindquist, S. L. The elongation of yeast prion fibers involves separable steps of association and conversion. Proc. Natl. Acad. Sci. U. S. A. 2004, 101 (8), 2287-2292.

(11) Paparcone, R.; Cranford, S. W.; Buehler, M. J. Self-folding and aggregation of amyloid nanofibrils. Nanoscale 2011, 3 (4), 1748-1755.

(12) Li, D.; Furukawa, H.; Deng, H.; Liu, C.; Yaghi, O. M.; Eisenberg, D. S. Designed amyloid fibers as materials for selective carbon dioxide capture. Proc. Natl. Acad. Sci. U. S. A. 2014, 111 (1), 191-196.

(13) Shimanovich, U.; Efimov, I.; Mason, T. O.; Flagmeier, P.; Buell, A. K.; Gedanken, A.; Linse, S.; Åkerfeldt, K. S.; Dobson, C. M.; Weitz, D. A. Protein Microgels from Amyloid Fibril Networks. ACS Nano 2015, 9 (1), 43-51.

(14) Jacob, R. S.; Ghosh, D.; Singh, P. K.; Basu, S. K.; Jha, N. N.; Das, S.; Sukul, P. K.; Patil, S.; Sathaye, S.; Kumar, A. Self healing hydrogels composed of amyloid nano fibrils for cell culture and stem cell differentiation. Biomaterials 2015, 54, 97-105.

(15) Cinar, G.; Ceylan, H.; Urel, M.; Erkal, T. S.; Deniz Tekin, E.; Tekinay, A. B.; Dâna, A.; Guler, M. O. Amyloid inspired self-assembled peptide nanofibers. Biomacromolecules 2012, 13 (10), 3377-3387.

(16) Krebs, M. R.; Bromley, E. H.; Donald, A. M. The binding of thioflavin-T to amyloid fibrils: localisation and implications. J. Struct. Biol. 2005, 149 (1), 30-37.

(17) Miti, T.; Mulaj, M.; Schmit, J. D.; Muschol, M. Stable, metastable, and kinetically trapped amyloid aggregate phases. Biomacromolecules 2015, 16 (1), 326-335.

(18) Streets, A. M.; Sourigues, Y.; Kopito, R. R.; Melki, R.; Quake, S. R. Simultaneous measurement of amyloid fibril formation by dynamic light scattering and fluorescence reveals complex aggregation kinetics. PLoS One 2013, 8 (1), e54541.

(19) Wördehoff, M. M.; Bannach, O.; Shaykhalishahi, H.; Kulawik, A.; Schiefer, S.; Willbold, D.; Hoyer, W.; Birkmann, E. Single Fibril Growth Kinetics of $\alpha$-Synuclein. J. Mol. Biol. 2015, 427 (6), 14281435.

(20) Chan, H.-M.; Xiao, L.; Yeung, K.-M.; Ho, S.-L.; Zhao, D.; Chan, W.-H.; Li, H.-W. Effect of surface-functionalized nanoparticles on the elongation phase of beta-amyloid (1-40) fibrillogenesis. Biomaterials 2012, 33 (18), 4443-4450.

(21) Guilbaud, J.-B.; Saiani, A. Using small angle scattering (SAS) to structurally characterise peptide and protein self-assembled materials. Chem. Soc. Rev. 2011, 40 (3), 1200-1210.

(22) Langkilde, A. E.; Vestergaard, B. Methods for structural characterization of prefibrillar intermediates and amyloid fibrils. FEBS Lett. 2009, 583 (16), 2600-2609.

(23) Bemporad, F.; Chiti, F. Protein misfolded oligomers: experimental approaches, mechanism of formation, and structuretoxicity relationships. Chem. Biol. 2012, 19 (3), 315-327.

(24) Kumaraswamy, P.; Sethuraman, S.; Krishnan, U. M. Hierarchical self-assembly of Tjernberg peptide at nanoscale. Soft Matter 2013, 9 (9), 2684-2694.

(25) Guimer, A.; Fournet, G. Small angle scattering of X-rays; J. Wiley \& Sons: New York, 1955.

(26) Pedersen, J. S.; Schurtenberger, P. Scattering functions of semiflexible polymers with and without excluded volume effects. Macromolecules 1996, 29 (23), 7602-7612.

(27) Chen, W.-R.; Butler, P. D.; Magid, L. J. Incorporating intermicellar interactions in the fitting of SANS data from cationic wormlike micelles. Langmuir 2006, 22 (15), 6539-6548.

(28) Niece, K. L.; Hartgerink, J. D.; Donners, J. J.; Stupp, S. I. Selfassembly combining two bioactive peptide-amphiphile molecules into nanofibers by electrostatic attraction. J. Am. Chem. Soc. 2003, 125 (24), $7146-7147$

(29) Xu, X.-D.; Chen, C.-S.; Lu, B.; Cheng, S.-X.; Zhang, X.-Z.; Zhuo, R.X. Coassembly of oppositely charged short peptides into welldefined supramolecular hydrogels. J. Phys. Chem. B 2010, 114 (7), 2365-2372.

(30) Takahashi, Y.; Ueno, A.; Mihara, H. Amyloid architecture: complementary assembly of heterogeneous combinations of three or four peptides into amyloid fibrils. ChemBioChem 2002, 3 (7), 637642.

(31) Ramachandran, S.; Trewhella, J.; Tseng, Y.; Yu, Y. B. Coassembling peptide-based biomaterials: effects of pairing equal and unequal chain length oligopeptides. Chem. Mater. 2006, 18 (26), 6157-6162.

(32) Swanekamp, R. J.; DiMaio, J. T.; Bowerman, C. J.; Nilsson, B. L. Coassembly of enantiomeric amphipathic peptides into amyloid- 
inspired rippled $\beta$-sheet fibrils. J. Am. Chem. Soc. 2012, 134 (12), 5556-5559.

(33) Makin, O. S.; Atkins, E.; Sikorski, P.; Johansson, J.; Serpell, L. C. Molecular basis for amyloid fibril formation and stability. Proc. Natl. Acad. Sci. U. S. A. 2005, 102 (2), 315-320.

(34) Jahn, T. R.; Makin, O. S.; Morris, K. L.; Marshall, K. E.; Tian, P.; Sikorski, P.; Serpell, L. C. The common architecture of cross- $\beta$ amyloid. J. Mol. Biol. 2010, 395 (4), 717-727.

(35) Gazit, E. A possible role for $\pi$-stacking in the self-assembly of amyloid fibrils. FASEB J. 2002, 16 (1), 77-83.

(36) Biancalana, M.; Koide, S. Molecular mechanism of Thioflavin-T binding to amyloid fibrils. Biochim. Biophys. Acta, Proteins Proteomics 2010, 1804 (7), 1405-1412.

(37) Nilsson, M. R. Techniques to study amyloid fibril formation in vitro. Methods 2004, 34 (1), 151-160.

(38) Hellstrand, E.; Boland, B.; Walsh, D. M.; Linse, S. Amyloid $\beta$ protein aggregation produces highly reproducible kinetic data and occurs by a two-phase process. ACS Chem. Neurosci. 2010, 1 (1), 1318.

(39) Meisl, G.; Yang, X.; Hellstrand, E.; Frohm, B.; Kirkegaard, J. B.; Cohen, S. I.; Dobson, C. M.; Linse, S.; Knowles, T. P. Differences in nucleation behavior underlie the contrasting aggregation kinetics of the $\mathrm{A} \beta 40$ and $\mathrm{A} \beta 42$ peptides. Proc. Natl. Acad. Sci. U. S. A. 2014, 111 (26), 9384-9389.

(40) Arosio, P.; Knowles, T. P.; Linse, S. On the lag phase in amyloid fibril formation. Phys. Chem. Chem. Phys. 2015, 17 (12), 7606-7618.

(41) Michaels, T. C.; Knowles, T. P. Kinetic theory of protein filament growth: Self-consistent methods and perturbative techniques. Int. J. Mod. Phys. B 2015, 29 (02), 1530002.

(42) Orbach, R.; Mironi-Harpaz, I.; Adler-Abramovich, L.; Mossou, E.; Mitchell, E. P.; Forsyth, V. T.; Gazit, E.; Seliktar, D. The rheological and structural properties of Fmoc-peptide-based hydrogels: the effect of aromatic molecular architecture on self-assembly and physical characteristics. Langmuir 2012, 28 (4), 2015-2022.

(43) Xu, F.; Li, J.; Jain, V.; Tu, R. S.; Huang, Q.; Nanda, V. Compositional control of higher order assembly using synthetic collagen peptides. J. Am. Chem. Soc. 2012, 134 (1), 47-50.

(44) Chatani, E.; Lee, Y.-H.; Yagi, H.; Yoshimura, Y.; Naiki, H.; Goto, Y. Ultrasonication-dependent production and breakdown lead to minimum-sized amyloid fibrils. Proc. Natl. Acad. Sci. U. S. A. 2009, 106 (27), 11119-11124.

(45) Kline, S. R. Reduction and analysis of SANS and USANS data using IGOR Pro. J. Appl. Crystallogr. 2006, 39 (6), 895-900.

(46) Enright, M. B.; Leitner, D. M. Mass fractal dimension and the compactness of proteins. Phys. Rev. E 2005, 71 (1), 011912.

(47) Koizumi, S.; Annaka, M.; Borbely, S.; Schwahn, D. Fractal structures of a poly ( $\mathrm{N}$-isopropylacrylamide) gel studied by small-angle neutron scattering over a $Q$-range from $10^{-5}$ to $0.1 \AA^{-1}$. Phys. B 2000, 276-278, 367-368. 